\title{
Actividad alimentaria de los peces herbívoros y su impacto en arrecifes con diferente nivel de degradación antrópica
}

\author{
Alain Durán ${ }^{1} \&$ Rodolfo Claro ${ }^{2}$ \\ 1, 2. Instituto de oceanología, Ministerio de Ciencia, Tecnología y Ambiente (CITMA), 1ra \# 18406, Rpto. Flores, Playa, \\ P.O. 12100, La Habana, Cuba, fax (537) 204 4355; alainduran@oceano.inf.cu; rclaro39@yahoo.es
}

Recibido 04-IV-2008. C Corregido 27-X-2008. Aceptado 29-XI-2008.

\begin{abstract}
The feeding activity of herbivorous fishes and their impact on coral reefs with different levels of anthropogenic degradation. The feeding activity of three herbivorous fishes (Sparisoma viride, Scarus iserti and Acanthurus coeruleus) and its impact on two coral reefs were studied during the dry (February) and rainy (August) seasons of 2006 in Cuba. One of the reef stations is at the "Acuario" dive point in the Guanahacabibes National Park, and is relatively pristine. The second one is located at the north coast of Havana City facing the Instituto de oceanología (IdO) and is subject to pollution and fishing pressures. Herbivore fish composition was analized, and foraging intensity was measured for all Acanthurid and Scarid species, by counting fish bites in $1 \mathrm{~m}^{2}$ during five minute intervals ( 25 replicates). Concurrently, species foraging intensity was measured for juvenile, intermediate, and adult phases, counting fish bites during 10 minutes intervals (10 replicates). Algal consumption rate was estimated using a coefficent of consumed biomass per bite, for each size class. The algal cover was sampled at $20 \mathrm{~m}$ linear transects with four replicates at each site. Food items were assessed sampling algae at the observed bitten substrates. Compared to Acuario, herbivores in IdO showed lower species richness and higher density of small size fishes, but large-sized parrotfish was almost absent due to intense fishing. The highest bite rate was observed for the smallest fish size, but net consumption rate was three times greater for medium and great size fishes, which were more abundant in the protected area. Algal cover was lower in Acuario, while in IdO it was very high, and coral cover was very low. In both locations epiphytic algae were the preferred food. These results support generalizations referring to the importance of great size herbivores fishes in controlling excesive algal proliferation on coral reefs. Rev. Biol. Trop. 57 (3): 687-697. Epub 2009 September 30 .
\end{abstract}

Key words: coral reefs, herbivore fishes, algal consumption; algal cover, Sparisoma viride, Scarus iserti, Acanthurus coeruleus, Guanahacabibes.

Algunos arrecifes del Caribe han sufrido un cambio de fase, de un estado dominado por corales a otro en que predominan las algas carnosas (Pandolfi et al. 2005). La actividad de los herbívoros, en particular los peces, juega un papel determinante en este proceso (Gardner et al. 2003, Pandolfi et al. 2003, Mumby 2006), sobre todo a partir de la disminución drástica de las poblaciones del erizo negro, Diadema antillarum (Hughes et al. 1999). Estos organismos generalmente consumen las algas pequeñas que forman el césped algal ("turf" en inglés; Paddack y Cowen 2006), lo que contribuye a evitar el excesivo crecimiento y expansión de las macroalgas en los arrecifes coralinos (Bellwood et al. 2004).

Como resultado del blanqueamiento y las enfermedades de los corales, estos están siendo sustituidos por las algas en muchos arrecifes (West y Salm 2003, Rogers y Millar 2006, Miller y Willians 2007) lo que, en algunos casos, se ha visto reforzado por la excesiva extracción pesquera de peces herbívoros (Mumby et al. 2006). Esto fue observado en la región norte-central de Cuba, donde ha disminuido la abundancia de peces herbívoros de 
mediana y gran talla por efecto de la pesca no sostenible, coincidiendo con el blanqueamiento masivo de corales y el aumento de la cobertura de algas (Claro et al. 2007).

Según Miller y Hay (1996) las condiciones anóxicas producidas por el enriquecimiento de nutrientes pueden deprimir la actividad alimentaria de los herbívoros, lo que corroboraron McClanahan et al. (2002) al encontrar una reducción de la intensidad de forrajeo en sitios con cobertura algal excesiva. Por otra parte, la actividad de los herbívoros también puede provocar efectos nocivos para la salud de los corales (McClanahan et al. 2005). Los peces loros (Scaridae) raspan la superficie de las rocas y de los corales para consumir algas y pólipos, lo que trae como consecuencia la reducción de la velocidad de crecimiento y la supervivencia de los corales, sobre todo después de los eventos de blanqueamiento (Rotjan et al. 2006).

Este trabajo tiene como objetivos caracterizar la actividad de búsqeuda de alimento de los peces herbívoros en dos arrecifes frontales con diferente grado de antropización y evaluar su influencia sobre la salud del arrecife, cuantificar la cobertura de algas en las dos localidades y su relación con la actividad alimentaria de los peces herbívoros, así como contribuir al conocimiento de la alimentación de tres de las especies más abundantes en Cuba y el Caribe.

\section{MATERIALES Y MÉTODOS}

Los muestreos se realizaron en dos arrecifes frontales fisiográficamente muy parecidos pero con muy diferente nivel de influencia antrópica. El primer sitio coincide con el punto de buceo conocido como Acuario ( $21^{\circ} 47.644^{\prime}$ $\left.\mathrm{N}-84^{\circ} 30.733^{\prime} \mathrm{W}\right)$, ubicado en el Parque nacional Península de Guanahacabibes, reconocido por su alto valor estético, la limpieza de sus aguas y el buen estado de conservación del arrecife coralino (Alcolado et al. 2003), por lo que es un importante atractivo turístico del Centro Internacional de Buceo María La Gorda. Esta área es poco accesible para la población, aunque existen evidencias de alguna pesca furtiva (Claro y Cantelar 2003). El estudio se realizó en una franja arrecifal paralela a la costa a una profundidad de cinco a ocho metros.

El segundo sitio de muestreo, ubicado cerca de la costa aledaña al Instituto de Oceanología (IdO; $23^{\circ} 05.678^{\prime} \mathrm{N}-82^{\circ} 28.769^{\prime} \mathrm{W}$ ), es un arrecife frontal al oeste de la ciudad de La Habana. El muestreo se realizó a lo largo de una franja arrecifal entre 8 y $11 \mathrm{~m}$ de profundidad. Este arrecife está fuertemente deteriorado por diversas fuentes de contaminación y una intensa pesca no comercial, debido al fácil acceso de la población (Aguilar y González-Sansón 2002, Aguilar 2005). Se encuentra muy cerca y al oeste del río Quibú, el cual vierte aguas muy contaminadas a la costa. Muy cerca existe además un emisario submarino cuya tubería ha sido dañada por el efecto mecánico de las olas durante las tormentas y libera aguas albañales con bajo nivel de tratamiento, cerca del sitio de observación. Los muestreos, en ambos sitios, fueron realizados en febrero (invierno) y agosto (verano), correspondientes con las épocas de seca y de lluvia, respectivamente.

Debido a la complejidad del estudio cualitativo y cuantitativo de la alimentación de los peces herbívoros a partir del contenido estomacal y por la inconveniencia de sacrificar un elevado número para ese fin, se utilizaron métodos no destructivos, que se describen a continuación. La intensidad de forrajeo y la densidad de los peces de las familias Scaridae y Acanthuridae se evaluaron mediante observación directa, utilizando el método descrito por Steneck (1983), que consiste en registrar la especie, talla estimada y número de mordiscos que realizan los peces herbívoros sobre un $1 \mathrm{~m}^{2}$ del sustrato durante 5 minutos, con un total de 25 réplicas en cada estación.

Para evaluar la intensidad de forrajeo a nivel de especie, se seleccionaron las tres más abundantes en Acuario, según Claro y Cantelar (2003): Sparisoma viride (Bonnaterre 1788), Scarus iserti (Bloch 1789) y Acanthurus coeruleus Bloch y Schneider 1801. Para cada especie se diferenciaron tres grupos de tallas: pequeños (5-10 $\mathrm{cm}$ de largo total $-\mathrm{LT})$, medianos $(10-15 \mathrm{~cm})$ y grandes $(>15 \mathrm{~cm})$. En el caso de $S$. viride, se utilizaron clases de 
talla mayores: para los medianos $(10-19 \mathrm{~cm})$ y grandes $(>19 \mathrm{~cm})$. Durante 10 minutos se contaron los mordiscos que realiza un pez sobre el sustrato. Se efectuaron diez conteos para cada uno de los tres intervalos de tallas en que se separaron los individuos de cada especie, para un total de 30 conteos por estación. Para disminuir la influencia del observador sobre la conducta del pez, se mantuvo una distancia de más de $2 \mathrm{~m}$. Se empleó la correlación de Pearson con un índice de confianza de .05 para comprobar si existía alguna relación entre la intensidad de forrajeo y la talla.

Para cuantificar el consumo de alimento, además del número de mordidas sobre el sustrato es necesario considerar la cantidad de alimento obtenido por estas, lo que depende del tamaño de la boca del pez. Para ello se utilizó el método descrito por Booth (1998): la densidad de cada una de las dos especies de loros (número de individuos por $\mathrm{m}^{2}$ ) se multiplicó por el número de mordiscos por día (extrapolando el promedio de mordidas en 10 minutos, a 10 horas diarias) y por la cantidad de materia vegetal consumida en cada mordida, según los coeficientes estimados por ese autor para cada clase de largos (iguales a las empleadas en este trabajo). Este experimento se realizó durante la época de lluvias.

La composición de la flora y la cobertura de algas se evaluaron empleando una modificación del método de transecto de intercepción lineal de Loya (1972), que consiste en registrar todos los géneros de algas observados y la longitud interceptada de la cubierta vegetal debajo de una cinta métrica de $20 \mathrm{~m}$ de largo, extendida sobre el arrecife, en cuatro posiciones paralelas a la costa en cada sitio.

Todos los géneros de algas presentes fueron ubicados en grupos morfofuncionales según Littler y Littler (1984) y Steneck y Dethier (1994). Dichos autores consideran como césped algal, a todas las algas con altura menor de $2 \mathrm{~cm}$.

Para estimar, aunque de forma indirecta, las preferencias alimentarias de los herbívoros estudiados, con una pinza se recolectaron las algas epífitas y otros organismos, presentes en el sitio justo donde se observó morder al pez. Se tomaron cinco muestras por cada clase de talla de cada una de las tres especies, en las dos localidades y épocas de muestreo. Estas muestras fueron fijadas en formol al 5\% para su posterior identificación.

Todos los análisis estadísticos fueron hechos con el programa SPSS 10, en su versión para Windows. Cuando los datos no cumplieron la normalidad se aplicó una transformación a logaritmo neperiano, como sucedió con el número de mordiscos sobre el sustrato. Para definir las diferencias significativas, se empleó ANOVA de clasificación simple. A los datos normales y homogéneos que dieron diferencias significativas se les aplicó una prueba StudentNewman-Keuls (SNK) a posteriori para conocer con mayor exactitud las medias que diferían. A los datos de número de mordidas, obtenidos con el método de Steneck (1983) se les aplicó una prueba Kruskal Wallis, no paramétrica, por no cumplir con las premisas para el ANOVA.

\section{RESULTADOS}

La riqueza de especies de peces herbívoros, fue mayor en la estación Acuario (ocho especies en período de lluvias y nueve en seca) que en IdO (cinco y seis, respectivamente), sin embargo, la densidad fue significativamente mayor en $\mathrm{IdO}\left(4 \pm 0.4\right.$ y $\left.5 \pm 0.5 \mathrm{ind} / \mathrm{m}^{2}\right)$ que en Acuario ( $3 \pm 0.2$ en ambos períodos; $\mathrm{p}<.05$ ). Así mismo, el número total de individuos fue mayor en IdO (Fig. 1). Las especies más abundantes en Acuario fueron $S$. viride y $A$. coeruleus, mientras que en IdO predominaron Sparisoma aurofrenatum (Valenciennes, 1840) y Acanthurus bahianus Castelnau, 1855. El loro listado, S. iserti, se encontró en ambos lugares, aunque fue más abundante en IdO. No se observaron diferencias en la composición por tallas de las especies más abundantes, entre las dos épocas del año en cada estación (Cuadro 1). Al unir las 10 especies de herbívoros encontradas en $\mathrm{IdO}$, se observó un predominio de peces de pequeña talla, y ninguno con más de $20 \mathrm{~cm} \mathrm{LT}$, mientras que en Acuario las tallas fueron superiores (Fig. 2). La talla promedio de $S$. viride 


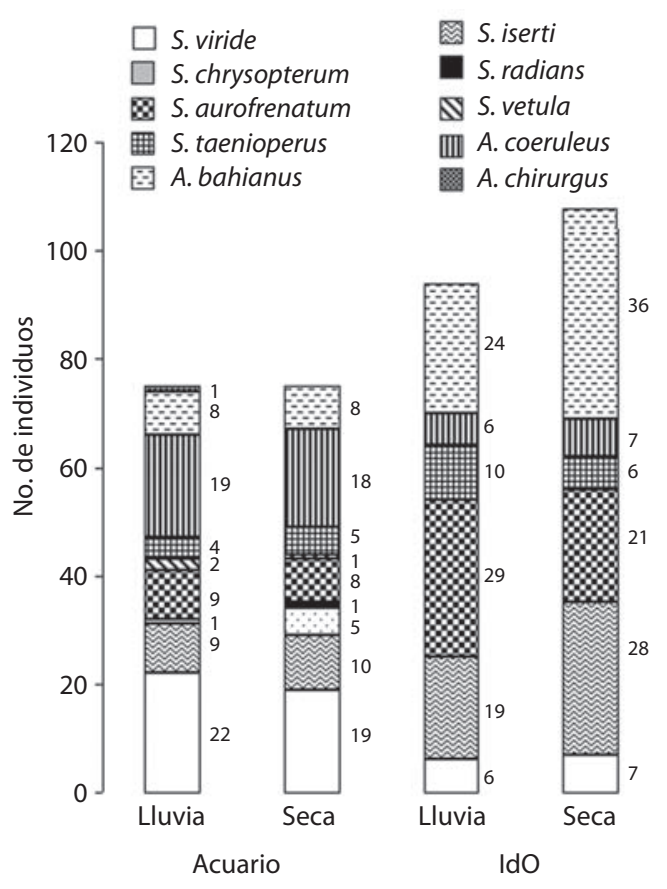

Fig. 1. Número total de peces herbívoros (barra completa) y número de individuos por especie (sectores de cada barra) en Acuario e IdO, en agosto (lluvia) y febrero (seca).

Fig. 1. Total number of herbivore fishes (full bar) and number of individuals per species (portion of each bar) at Acuario and IdO, in August (rainy season) and February (dry season).

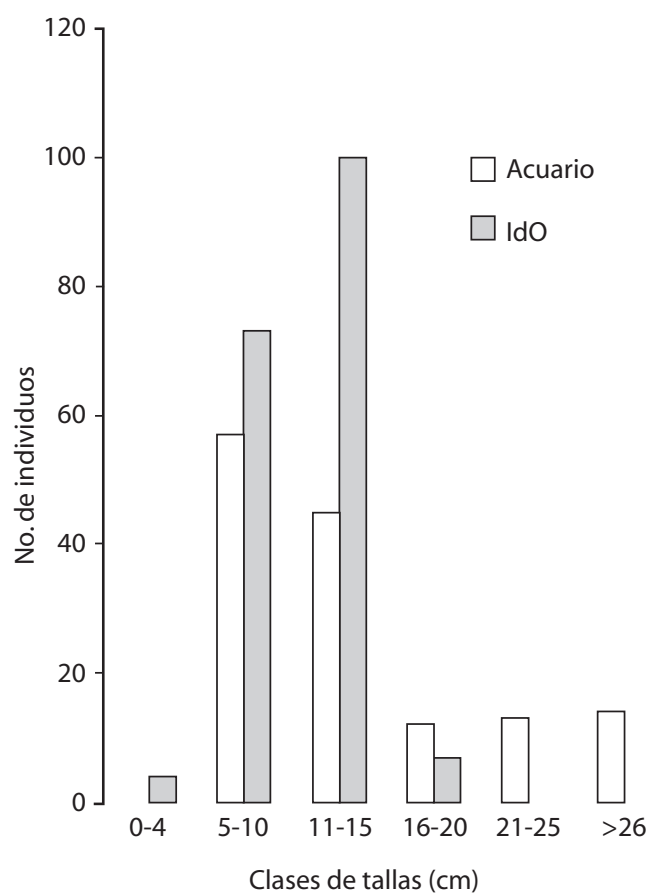

Fig. 2. Composición por tallas de los peces herbívoros observados en Acuario e IdO.

Fig. 2. Size composition of herbivore fishes observed at Acuario and $\mathrm{IdO}$.

\section{CUADRO 1}

Especies de peces herbivoros más frecuentes, número de individuos y talla promedio en los arrecifes frontales de Acuario e Instituto de oceanología

TABLE 1

More frequent herbivore fish species, individuals number and average size at fore reefs of Acuario and Instituto de oceanología

\begin{tabular}{|c|c|c|c|c|}
\hline \multirow[t]{2}{*}{ Especies } & No. Ind. & Talla \pm EE (cm) & No. Ind. & Talla \pm EE $(\mathrm{cm})$ \\
\hline & \multicolumn{2}{|c|}{ Acuario-agosto } & \multicolumn{2}{|c|}{ Acuario-febrero } \\
\hline Sparisoma viride & 22 & $19.7 \pm 2.2$ & 19 & $19.4 \pm 1.3$ \\
\hline Scarus iserti & 9 & $10.1 \pm 0.9$ & 10 & $11.4 \pm 0.9$ \\
\hline \multirow[t]{2}{*}{ Acanthurus coeruleus } & 19 & $11.0 \pm 0.7$ & 18 & $10.3 \pm 0.5$ \\
\hline & \multicolumn{2}{|c|}{ IdO-agosto } & \multicolumn{2}{|c|}{ IdO-febrero } \\
\hline Sparisoma aurofrenatum & 29 & $12.6 \pm 1.4$ & 21 & $7.2 \pm 1.3$ \\
\hline Scarus iserti & 18 & $12.7 \pm 0.3$ & 27 & $12.1 \pm 0.4$ \\
\hline Acanthurus bahianus & 24 & $11.6 \pm 1.1$ & 36 & $6.8 \pm 1.0$ \\
\hline
\end{tabular}

$\mathrm{EE}=$ error estándar. 
fue mayor en Acuario que en IdO, en ambas épocas de muestreo.

La intensidad de forrajeo de $S$. iserti y $A$. coeruleus, fue similar (Fig. 3), mientras que la de $S$. viride fue significativamente menor ( $\mathrm{p}<$ .001). Las tres especies analizadas mostraron menor actividad alimentaria en el período de seca-invierno La diferencia fue significativa para $S$. viride $(\mathrm{p}<.001)$ y S. iserti $(\mathrm{p}<.05)$, pero no para A. coeruleus (Fig. 3). Sin embargo, la intensidad de mordisqueo de las tres especies fue similar en los dos sitios.

La actividad alimentaria, expresada por el número de mordiscos sobre el sustrato, fue menor en los peces de mayores tallas de las tres especies. Aunque los coeficientes de correlación negativa entre ambas variables fueron bajos, el análisis SNK mostró diferencias entre los grupos de tallas (Cuadro 2).

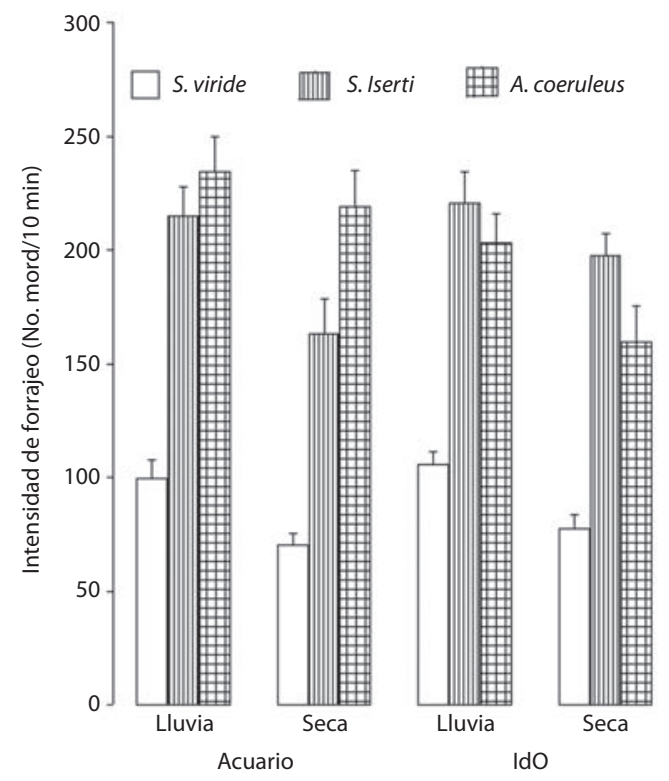

Fig. 3. Intensidad de forrajeo de tres especies de peces herbívoros, estimada con base en el número promedio de mordiscos sobre el sustrato. Las líneas verticales indican el error estándar.

Fig. 3. Grazing rate of three species of herbivore fishes, estimated from the average number of bites on the substrate. Vertical lines indicate the Standard error.
La comparación del consumo por clases de tallas mostró que, en Acuario, el número de mordiscos sobre el sustrato por los peces menores de $10 \mathrm{~cm}$ fue mucho más alto, pero el consumo estimado para los de más de $20 \mathrm{~cm}$, fue 28 veces mayor. En IdO, sin embargo, debido a la baja densidad de peces de tallas grandes, el consumo por parte de los individuos pequeños fue mayor, aunque el consumo total de algas fue tres y media veces menor que en Acuario. En IdO, predominaron $S$. iserti (cuyo tamaño máximo es generalmente inferior a $10 \mathrm{~cm} \mathrm{LT}$ ), ejemplares pequeños de $S$. viride, y en general muy baja densidad de herbívoros mayores de $15 \mathrm{~cm}$, mientras que en Acuario se observó una densidad similar de peces de las tres clases de tallas (Cuadro 3).

Aunque el número de géneros de algas encontrados fue similar en las dos localidades, tanto en agosto (13 y 17 géneros en Acuario e IdO respectivamente) como en febrero ( 9 y 10 géneros), el cubrimiento total por macroalgas fue mayor en IdO ( $p<.01$; Fig. 4$)$ y presentó los valores más altos en febrero $(\mathrm{p}<.05)$.

La cobertura del césped algal en Acuario, durante el período de seca, fue el doble que en el de lluvia $(\mathrm{p}<.001)$, pero no se observó esa diferencia en IdO. Entre las macroalgas coriáceas, Lobophora variegata (única especie del género registrada en Cuba; Suárez 2005) fue la más abundante. Este grupo morfofuncional presentó mayor cobertura en IdO que en Acuario $(\mathrm{p}<.01)$ y hubo diferencias entre las épocas en los dos lugares $(p<.05$ en ambos sitios), aunque tales diferencias no parecen ser estacionales, ya que en Acuario se observó mayor cobertura de este grupo en la época de lluvia, mientras que en IdO fue ocho veces mayor en la época de seca.

Las macroalgas foliosas-globosas estuvieron representadas casi en su totalidad por el género Dictyota y su cobertura fue significativamente mayor en Acuario $(p<.05)$. No se encontraron diferencias significativas entre las épocas en ninguno de los dos sitios Las macroalgas calcáreas presentaron porcentajes bajos de cobertura, solo se observó mayor porcentaje en IdO, en el mes de seca $(\mathrm{p}<.01)$. 
CUADRO 2

Número promedio de mordiscos sobre el sustrato, por intervalos de tallas, de tres especies de herbivoros

TABLE 2

Mean number of bites on the substrate, per size classes of three herbivorous fishes

\begin{tabular}{lccccc} 
Especies & \multicolumn{2}{c}{ Número promedio de mordiscos (EE) } & ANOVA & $\begin{array}{c}\text { Coeficiente de } \\
\text { correlación } \\
\text { Pearson }\end{array}$ \\
& Pequeños & Medianos & Grandes & $(\mathrm{F})$ & $-0.356(\mathrm{p}>.01)$ \\
Sparisoma viride & $104.1 \pm 6.9 \mathrm{~b}$ & $86.3 \pm 4.6 \mathrm{a}$ & $72.3 \pm 4.7 \mathrm{a}$ & 8.5 & $-0.211(\mathrm{p}>.05)$ \\
Scarus iserti & $209.1 \pm 12.7 \mathrm{a}$ & $212.4 \pm 11.4 \mathrm{~b}$ & $170.8 \pm 10.2 \mathrm{a}$ & 4.0 & $-0.356(\mathrm{p}>.01)$ \\
Acanthurus coeruleus & $240.3 \pm 13.4 \mathrm{~b}$ & $205.9 \pm 14.1 \mathrm{~b}$ & $165.6 \pm 10.7 \mathrm{a}$ & 8.5 & -10.5
\end{tabular}

Las letras indican el resultado del análisis SNK "a posterior" $(\mathrm{p}<.05) . \mathrm{N}=30$ en todas las clases de tallas.

The letters show the results of the SNK test "a posteriori" $(\mathrm{p}<.05) . \mathrm{N}=30$ for all size classes.

$\mathrm{EE}=$ error estándar.

\section{CUADRO 3}

Consumo de algas estimado para dos especies de loros y variables utilizadas para los cálculos

TABLE 3

Estimated algal consumption by two parrotfish species, and variables used for calculations

\begin{tabular}{|c|c|c|c|c|}
\hline $\begin{array}{l}\text { Grupos de tallas } \\
(\mathrm{cm})\end{array}$ & $\begin{array}{l}\text { No. promedio de } \\
\text { mordiscos/día } \\
\text { (10 horas })\end{array}$ & $\begin{array}{c}\text { Densidad } \\
\text { de peces } \\
\left(\text { No ind } / \mathrm{m}^{2}\right)\end{array}$ & $\begin{array}{c}\text { Consumo } \\
\text { por mordida. } \\
(\mathrm{mg} \text { PS/mordisco)* }\end{array}$ & $\begin{array}{l}\text { Consumo total } \\
\text { (g PS } / \mathrm{m}^{2} / \text { día) }\end{array}$ \\
\hline \multicolumn{5}{|l|}{ Acuario } \\
\hline Pequeños (6-10) & 10158 & 0.55 & 0.05 & 0.3 \\
\hline Medianos (10-19) & 9090 & 0.56 & 0.5 & 2.1 \\
\hline Grandes $(>20)$ & 7860 & 0.54 & 2 & 8.5 \\
\hline Total & & & & 10.9 \\
\hline \multicolumn{5}{|l|}{$\mathrm{IdO}$} \\
\hline Pequeños (6-10) & 10110 & 1.50 & 0.05 & 0.8 \\
\hline Medianos (10-19) & 11397 & 0.35 & 0.5 & 2.0 \\
\hline Grandes $(>20)$ & 7839 & 0.02 & 2 & 0.3 \\
\hline Total & & & & 3.1 \\
\hline
\end{tabular}

Las macroalgas corticadas fueron escasas en ambos sitios.

En las muestras obtenidas en los sustratos de mordisqueo, de las tres especies de herbívoros estudiados se identificaron 58 géneros de algas, distribuidos en los siguientes grupos morfofuncionales: 29 filamentosas, 10 foliosas y globosas, 8 corticadas, 5 calcáreas articuladas,
2 costrosas, 2 calcáreas y 2 coriáceas. Asociadas a estas algas se encontraron cianobacterias, y colonias de hidrozoos y foraminíferos, los que también son consumidos, al parecer de forma incidental.

La mayor actividad de mordisqueo se observó sobre las algas filamentosas, las que estuvieron presentes en la totalidad de las 


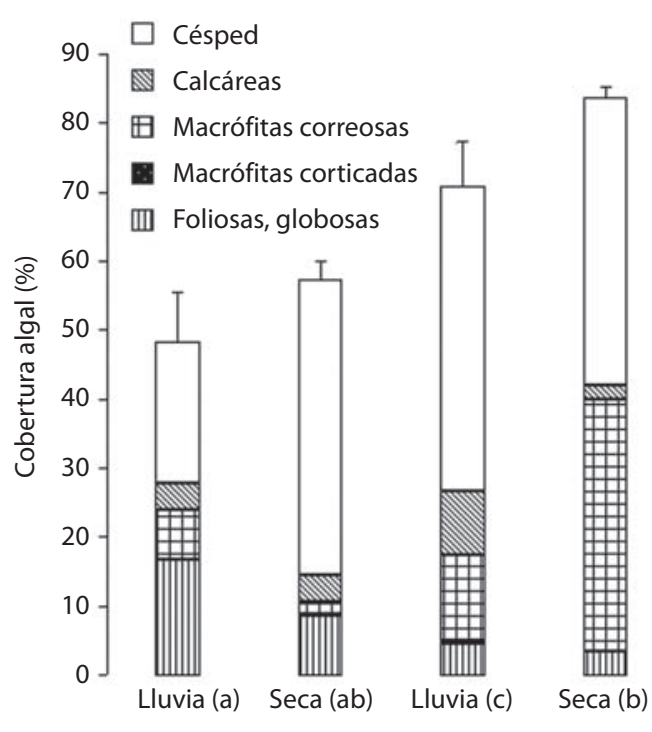

Fig. 4. Cobertura algal por grupos morfofuncionales en Acuario e IdO, en dos épocas del año. Las letras entre paréntesis indican los resultados de la prueba SNK; las líneas verticales, el estándar de la cobertura total.

Fig. 4. Macroalgal cover by morfofunctional groups at Acuario and IdO, in two seasons of the year. The letters in parenthesis indicate the results of SNK test; vertical lines -the standard error of total cover. muestras (Cuadro 4), Los géneros Jania y Dictiota, fueron segundos por su frecuencia en los sustratos de alimentación de las tres clases de talla, de S. iserti y A. coeruleus (Cuadro 5). Solo los individuos pequeños de $S$. viride no mostraron actividad sobre el género Dictyota. Estos concentraron su actividad de forrajeo sobre las algas filamentosas (con excepción de Ceramium).

\section{DISCUSIÓN}

La existencia de menor riqueza de especies y menor densidad de herbívoros de mediana y gran talla en IdO, parece ser consecuencia del alto nivel de estrés en el área, principalmente por la presión de pesca. Según Aguilar (2005), esa área costera se encuentra altamente afectada por la pesca no comercial y dominada por especies de pequeña talla, de bajo nivel trófico y ausencia casi total de especies de alto valor comercial. Por otra parte, dicha área costera presenta niveles altos de nitratos, nitritos y fosfatos (Montalvo et al. 1998) característicos de aguas de mala calidad según la Norma Cubana NC- 25:99 (1999). Esta contaminación

\section{CUADRO 4}

Organismos con frecuencia de aparición mayor que $10 \%$ en los sustratos de mordisqueo de tres especies de peces herbivoros

TABLE 4

Organisms with occurrence frequency higher than $10 \%$ on bitten substrates by three herbivore fish

$\begin{array}{llc}\text { Géneros de algas y otros alimentos } & \text { Grupos morfo-funcionales } & \text { Frecuencia (\%) } \\ \text { Dictyota } & \text { Foliosas-globosas } & 47.2 \\ \text { Jania } & \text { Calcáreas-articuladas } & 42.2 \\ \text { Ceramiun } & \text { Filamentosas } & 35.6 \\ \text { Cianobacterias } & - & 28.3 \\ \text { Gelidiella } & \text { Corticadas } & 17.2 \\ \text { Polysiphonia } & \text { Filamentosas } & 16.7 \\ \text { Lobophora } & \text { Coriáceas } & 15.6 \\ \text { Foraminíferos } & - & 13.3 \\ \text { Herposiphonia } & \text { Filamentosas } & 12.2 \\ \text { Gelidium } & \text { Filamentosas } & 10.0\end{array}$


CUADRO 5

Géneros de algas con frecuencia de aparición mayor del $25 \%$ en los sustratos de forrajeo de tres especies de herbívoros, por clases de tallas

TABLE 5

Algae genera with occurrence frequency higher than $25 \%$ at forage substrates of three herbivore fishes, by size class

\begin{tabular}{|c|c|c|c|c|c|c|}
\hline \multirow{3}{*}{ Tallas } & \multirow{2}{*}{\multicolumn{2}{|c|}{ S. viride }} & \multirow{2}{*}{\multicolumn{2}{|c|}{ S. iserti }} & \multirow{2}{*}{\multicolumn{2}{|c|}{ A. coeruleus }} \\
\hline & & & & & & \\
\hline & Géneros & $\mathrm{F}(\%)$ & Géneros & $\mathrm{F}(\%)$ & Géneros & $\mathrm{F}(\%)$ \\
\hline \multirow{4}{*}{ Grandes } & Jania & 60 & Jania & 45 & Jania & 35 \\
\hline & Dictyota & 40 & Dictyota & 30 & Dictyota & 40 \\
\hline & Ceramium & 25 & Ceramium & 35 & Ceramium & 40 \\
\hline & - & & Cianobacterias & 35 & Cianobacterias & 25 \\
\hline \multirow{4}{*}{ Medianas } & Jania & 30 & Jania & 35 & Jania & 35 \\
\hline & Gelidium & 35 & Dictyota & 50 & Dictyota & 55 \\
\hline & Dasya & 30 & Ceramium & 35 & Ceramium & 40 \\
\hline & - & & Cianobacterias & 30 & Polysiphonia & 35 \\
\hline \multirow{4}{*}{ Pequeñas } & Gelidiella & 50 & Jania & 50 & Dictyota & 25 \\
\hline & Flahayaultia & 25 & Dictyota & 60 & Ceramium & 45 \\
\hline & Haliptilon & 25 & Ceramium & 55 & Cianobacterias & 50 \\
\hline & - & & Cianobacterias & 30 & Gelidiella & 25 \\
\hline
\end{tabular}

influye de forma directa en el deterioro de la ictiofauna (Aguilar 2005). Klomp et al. (2003) observaron bajas biomasas de peces herbívoros en zonas fuertemente antropizadas del Caribe y ausencia casi total de individuos adultos de $S$. viride. Estos autores observaron una tendencia a la reducción de la variación genética y un menor potencial reproductivo asociado a menores tallas de reproducción. También como resultado de una alta presión de pesca en los arrecifes de Jamaica, Hawkins y Roberts (2003) concluyeron que el reclutamiento de los herbívoros en el área depende de la importación de larvas desde sitios alejados y mejor conservados. Sin embargo, tanto en Acuario como en IdO, el reclutamiento no parece ser un factor limitante, a juzgar por la riqueza de especies y abundancia de peces juveniles que observamos.

Por las características de su aparato bucal, $S$. viride pertenece al grupo de los "raspadores" (scrappers) que presentan un alto nivel de asimilación de alimentos (Bruggemann et al. 1994a); lo que explica su menor frecuencia de mordisqueo en comparación con $A$. coeruleus. La alta frecuencia de mordisqueo de S. iserti, que es un raspador también, se debe, al parecer, a la necesidad de compensar la baja eficiencia de una boca pequeña con una mayor actividad (Bruggemann et al. 1994b; Booth 1998, Fox y Bellwood 2007). La menor actividad alimentaria durante el período invernal puede ser consecuencia de la reducción del metabolismo, provocado por la disminución de la temperatura del agua (Klumpp y Polunin 1989, Hay y Steinberg 1992, Koop et al. 2001).

En IdO, como resultado de la pesca intensiva de los herbívoros, predominaron $S$. iserti (cuya talla máxima es generalmente inferior a $10 \mathrm{~cm} \mathrm{LT),} \mathrm{ejemplares} \mathrm{pequeños} \mathrm{de} S$. viride, y en general muy baja densidad de herbívoros de tallas mayores de $15 \mathrm{~cm}$, mientras que en Acuario, donde prácticamente no se pescan los herbívoros, se observó una densidad similar de peces de las tres clases de tallas. 
El alto consumo de algas por los herbívoros de mayor talla, observado en Acuario, se evidenció como un factor importante en el control del sobrecrecimiento algal, mientras que la ausencia de herbívoros grandes en IdO y, en consecuencia, el bajo nivel de consumo total, coincidió con un elevado nivel de cobertura de algas. Tales resultados sugieren la necesidad de mantener una adecuada estructura de tallas en las poblaciones de herbívoros, la que puede ser seriamente sesgada por la pesca, como se observa en el caso de la estación IdO. Este mismo factor es causa de una alta densidad de herbívoros de pequeña talla, los que por una parte, como reafirman nuestros resultados, son menos eficientes en el control de las algas, y por otra, producen mayor número de mordiscos sobre los corales, lo que podría contribuir al deterioro de estos.

Gracias a la existencia de una estructura de tallas cercana a la natural en el arrecife protegido, la presión de forrajeo expresada por la intensidad de consumo estimada por unidad de área en el mismo, fue tres veces mayor, que en el sometido a una pesca indiscriminada y tensionado por la contaminación.

La alta frecuencia de los géneros Dyctiota y Jania en los sustratos de mordisqueo, parece contradictoria ya que, las primeras contienen diterpenos repelentes para los herbívoros (Barbosa et al. 2004), y las segundas son calcáreas y por tanto muy poco consumidas por los herbívoros (Littler et al. 1983, Steneck y Dethier 1994). Sin embargo, las algas de esos géneros y además las de Lobophora, se caracterizaron por un alto nivel de epifitismo. Al parecer, estos herbívoros son capaces de consumir solo las epífitas, sin ingerir las algas sobre las cuales éstas se desarrollan, o no ser afectados por el efecto repelente de las tóxicas.

La preferencia por las algas filamentosas coincide con los resultados de otros autores (Lewis 1986, Steneck y Dethier 1994). Peirano et al. (2001) observaron que los peces herbívoros prefieren las hojas de Thalassia con mayor cobertura de epífitas y contenido de nitrógeno. Bruggemann et al. (1994b) observaron en un arrecife, que el $95 \%$ de las mordidas de
S. viride fueron sobre las algas filamentosas que crecían encima de esqueletos de corales muertos. Según estos autores, el consumo de estas algas, que son las de mayor contenido proteico, les confiere una ventaja adaptativa a esta especie.

La escasez de peces herbívoros de mediana y gran talla en IdO, debido a la alta presión pesquera (Aguilar 2005), contribuye a una mayor cobertura algal en esta zona (ya de por sí estimulada por el aporte de nutrientes) y por tanto, a una disminución de la cubierta coralina y de la complejidad estructural del arrecife.

\section{AGRADECIMIENTOS}

Agradecemos el apoyo logístico ofrecido por el Instituto de Oceanología, el Centro de Investigaciones Marinas de la Universidad de La Habana, y el Centro Internacional de Buceo María La Gorda, para la ejecución de la investigación. A Pedro Alcolado y Beatriz Martínez por sus sugerencias y criterios en la preparación del manuscrito, a Macario Esquivel por la identificación de las algas, y a los técnicos y buzos que colaboraron en los muestreos, Miguel Hernández, Jorge Luís Hernández, Rogelio y Rolando Días y Pedro Alcolado (hijo).

\section{RESUMEN}

Estudiamos la actividad alimentaria de tres peces herbívoros (Sparisoma viride, Scarus iserti y Acanthurus coeruleus) y su impacto, en dos arrecifes de coral de Cuba, durante la época seca (febrero) y la lluviosa (agosto) del 2006. Una de las estaciones en los arrecifes está en el lugar de buceo "Acuario" en el parque nacional Guanahacabibes, y es relativamente prístina. La segunda está en la costa norte de la Habana al frente del Instituto de oceanología (IdO) y es objeto de presiones de contaminación y pesca. La composición de los peces herbívoros fue analizada, y la intensidad de búsqueda de alimento ("forrajeo") medida para todas las especies de acantúridos y escáridos, contando los mordiscos de peces en $1 \mathrm{~m}^{2}$ durante intervalos de cinco minutos (25 repeticiones). La intensidad de forrajeo fue medida para las fases joven, intermedia, y adulta, contando los mordiscos de peces durante intervalos de 10 minutos (10 repeticiones). La tasa de consumo de algas se estimó usando un coeficiente de biomasa consumida por mordisco, para cada clase de tamaño. La cobertura de algas fue muestreada en transectos de $20 \mathrm{~m}$ en línea con cuatro 
repeticiones en cada sitio. Las piezas de comida fueron evaluadas por medio de muestreo de algas en los sustratos observados con mordiscos. En comparación con Acuario, los herbívoros en $\mathrm{IdO}$ mostraron riqueza de especies más baja y mayor densidad de peces pequeños, pero los peces loro de gran tamaño estuvieron casi ausentes debido a la pesca intensiva. La tasa de mordisqueo más alta se observó para los peces de tamaño más pequeño, pero la tasa neta de consumo fue tres veces mayor para los peces de mediano y gran tamaño, los cuales fueron más abundantes en el área protegida. La cobertura de algas fue más baja en Acuario, mientras que en IdO fue muy alta, y la cobertura de coral fue muy baja. En ambas localidades las algas epífitas fueron el alimento preferido. Estos resultados apoyan las generalizaciones con respecto a la importancia de los peces de gran tamaño para controlar la proliferación excesiva de algas en arrecifes de coral.

Palabras clave: coral reefs, herbivore fishes, algal consumption; algal cover, Sparisoma viride, Scarus iserti, Acanthurus coeruleus, Guanahacabibes.

\section{REFERENCIAS}

Aguilar, B. C. 2005. La ictiofauna de la Ciudad Habana: Efectos acumulativos de agentes estresantes múltiples en varios niveles de organización biológica. Tesis de Doctorado. Centro de Investigaciones Marinas, Universidad de La Habana, Cuba. 122 p.

Aguilar, C. y G. González-Sansón. 2002. Ecología de la ictiofauna costera en la zona adyacente a la desembocadura del río Almendares (La Habana, Cuba). 1. Distribución espacial de la abundancia y la diversidad. Rev. Invest. Mar. 23(1): 3-14.

Alcolado, M. P., B. Martínez-Daranas, G. MenéndezMacía, R. del Valle, M. Hernández y T. García. 2003. Rapid assessment of coral communities of María la Gorda, southeast Ensenada de Corrientes, Cuba (Part 1: stony corals and algae), p. 268-277. En J.C. Lang, (ed.). Status of Coral Reefs in the Western Atlantic: Results of initial surveys, Atlantic and Gulf Rapid Reef Assessment (AGRRA) Program. Atoll Research Bulletin: 496: 268-277.

Barbosa, J. P., V. L.Teixeira y R. C. Pereira. 2004. A dolabellane diterpene from the brown alga Dictyota pfaffii as chemical defense against herbivores. Bot. Mar. 47(2): 147-151.

Bellwood, D. R., T. P. Hughes, C. Folke y M. Nystrom. 2004. Confronting the coral reef crisis? Nature. 429: 827- 833 .

Booth, D. J. 1998. Grazing pressure of roving and territorial fishes on patch reef in One Tree Lagoon.
Proc. Australian Coral Reef Society $75^{\text {th }}$ Anniversary Conference: 15-27.

Bruggemann, J. H., J. Begeman, E. M. Bosma, P. Verburg y A.M. Breeman. 1994a. Foraging by the stoplight parrotfish Sparisoma viride. II. Intake and assimilation of food. Protein and energy. Mar. Ecol. Prog. Ser. 106: 57-71.

Bruggemann, J. H., M. J. H. Van Oppen y A. M. Breeman. 1994b. Foraging by the stoplight parrotfish Sparisoma viride. I. Food selection in different, socially determined habitats. Mar. Ecol. Prog. Ser. 106: 41-55.

Choat, J. H. y K. D. Clements. 1993. Daily feeding rates in herbivorous labroid fishes. Mar. Biol. 117: 205-211.

Claro, R.y K. Cantelar. 2003. Rapid assessment of the coral communities of María La Gorda, Southeast Ensenada de Corrientes, Cuba (Part 2: reef fishes). Atoll Res. Bull 496: 278-293.

Claro, R., K. Cantelar, F. Pina Amargós y J. P. GarcíaArteaga. 2007. Cambios en las comunidades de peces de los arrecifes coralinos del Archipiélago SabanaCamagüey, Cuba. Rev. Biol. Trop. 55 (2): 537-547.

Fox, R. J. y D. R. Bellwood. 2007. Quantifying herbivory across a coral reef depth gradient. Mar. Ecol. Prog. Ser. 339: 49-59.

Gardner, T. A., I. M. Cote, J. A. Gill, A. Grant y A. R. Watkinson. 2003. Long-term region-wide declines in Caribbean corals. Science. 301: 958-960.

Hawkins, J. P. y M. C. Roberts. 2003. Effects of fishing on sex-changing Caribbean parrotfishes. Biol. Conserv. 115: 213-226.

Hay, M. E y P. D. Steinberg. 1992. The chemical ecology of plant-herbivore interactions in marine versus terrestial Communities, pp. 371-413. En G. A. Rosenthal y M. R. Berenbaum (eds). Marine and terrestrial Chemical Ecology. Academic Press. Inc.

Hughes, T. P., A. M. Szmant, R. Steneck, R. Carpenter y S. Miller. 1999. Algal blooms on coral reefs: what are the causes? Limnol Oceanogr. 44: 1583-1586.

Klomp, K. D., K. Clarke, K. Marks y M. Miller. 2003. Condition of reef fish on Jamaica's north coast signals late stages of overexploitation. Proc. Gulf. Caribb. Fish. Inst. 54: 799-803.

Klumpp, D. M y N. V. C. Polunin. 1989. Partitioning among grazers of food resources within damselfish territories on a coral reef. J. Exp. Mar. Biol. Ecol. 125: $145-169$. 
Koop, K., D. Booth, A. Broadbent, J. Bucher, D. Capone, J. Coll, W. Dennison, M. Erdman, P. Harrison, O. Hoegh-Gulderg, P. Hutchings, G. B. Jones, A. W. D. Larkum, J. O’Neil, A. Steven, E. Tentori, S. Ward, J. Williamson y D. Yellowlees. 2001. ENCORE: The effect of nutrient enrichment on coral reefs, synthesis of results and conclusions. Mar. Pollut. Bull. 42 (2): 91-120.

Lewis, S. M. 1986. The role of herbivorous fishes in the organization of the Caribean reef community. Ecol. Monogr. 56 (3): 183-200.

Littler, M. M y D. S. Littler. 1984. Models of Tropical Reef Biogenesis. The contribution of algae, pp. 323364 En: F. E. Round y D. J. Chapman (eds.), Progr. Phycol. Research 3. Biopress, Bristol.

Littler, M., P. R. Taylor y D. S. Littler. 1983. Algal resistance to herbivory on a Caribbean Barrier Reef. Coral Reefs. 2: 111-118.

Loya, Y. 1972. Community structures and fishes diversity of hermatypic corals at Eilat, Red Sea. Mar. Biol. 13(2): $100-123$

McClanahan, T. R, B. A. Cokos y E. Sala. 2002. Algal growth and species composition under experimental control of herbivory, phosphorus and coral abundance in Glovers Reef, Belize. Mar. Pollut. Bull. 44 (6): 441-451.

McClanahan, T. R., J. Maina, C. J. Starger, P. Herron-Perez y E. Dusek. 2005. Detriments to post-bleaching recovery of corals. Coral Reefs. 24: 230-246.

Miller, M. W. y D. E. Willians. 2007. Coral disease outbreak at Navassa, a remote Caribbean Island. Coral Reefs. 26: 97-101.

Miller, M. W. y M. E. Hay. 1996. Coral-seaweed-grazernutrient interactions on temperate reef. Ecol. Monog. 66: $323-344$

Montalvo, J. F., M. Cano, E. Perigó, S. Esponda, E.M. Cesar y C.M. Peón. 1998. Evaluación de factores hidroquímicos relacionados con la contaminación orgánica en tres cuerpos costeros del litoral oeste de Ciudad de la Habana, pp. 93-97. En F. Martínez, L. Sorinas, O. Arjona, J. Estévez y J. Santana (eds.), Contribución a la educación y protección ambiental. Editorial Academia, La Habana.

Mumby, J. P. 2006. The impact of exploiting grazers (scaridae) on the dynamics of Caribbean coral reefs. Ecol. Appl. 16(2): 747-769.

Mumby, P. J., C. P. Dahlgren, A. R. Harbone, C. V. Kappel, F. Micheli, D. R. Brumbaugh, K. E. Holmes, J. M.
Mendes, K. Broad, J. N. Sanchirico, K. Buch, S. Box, R. W. Stoffle y A. B. Gill. 2006. Fishing, trophic cascades, and the process of grazing on coral reffs. Science. 311: 98-101.

Norma Cubana (NC:25:99). 1999. Sistema de Normas para la Protección del Ambiente. Hidrósfera. Especificaciones y procedimientos para la evaluación de los objetos hídricos de uso pesquero. La Habana, Cuba, 12 p.

Paddack, M. J. y R. K. Cowen. 2006. Grazing pressure of the herbivorous fishes on coral-cover reefs. Coral Reefs. 25: 461-472.

Pandolfi, J. M., J. B. C. Jackson, N. Baron, R. H. Bradbury, H. M. Guzman, T. P. Hughes, C. V. Kappel, F. Micheli, J. C. Ogden, H. P. Possingham y E. Sala. 2005. Are U.S. coral reefs on the slippery slope to slime? Science. 37: 1725-1726.

Pandolfi, J. M., R. H. Bradbury, E. Sala, T. P. Hughes, K. A. Bjorndal, R. G. Cooke, D. McArdle, L. McClenachan, M. Newman, G. Paredes, R. R. Warner y J. B. C. Jackson. 2003. Global trajectories of the long-term decline of coral reef ecosystems. Science 301:955-958

Peirano, A., I. Niccolai, R. Mauro y C. N. Bianchi. 2001. Seasonal grazing and food preference of herbivores in a Posidonia oceanica meadow. Sci. Mar. 65(4): 367-374.

Rogers, C. S. y J. Millar. 2006. Permanent phase shifts or reversible declines in coral cover? Lack of recovery of two coral reefs in St John, Virgin Islands. Mar. Ecol. Prog. Ser. 306: 103-114.

Rotjan, R. D., J. L. Dimond, D. J. Thornhill, J. J. Leichter, B. Helmuth, D. W. Kemp y S. M. Lewis. 2006. Chronic parrotfish grazing impedes coral recovery after bleaching. Coral Reefs. 25: 361-368.

Steneck, R. S y M. N. Dethier. 1994. A functional group approach to the structure of algal-dominated communities. Oikos 69: 476-498.

Steneck, R. S. 1983. Quantifying herbivory on coral reefs: Just scratching the surface and still biting off more than we can chew. En L. Reaka (ed.). The ecology of deep and shallow coral reefs, Simposia Series for Undersea Reserarch, NOAA'S Undersea Research Program. 1(1): 103-111.

Suárez, A. M. 2005. Lista de macroalgas marinas de Cuba. Rev. Invest. Mar. 26(2): 93-148.

West, J. M. y R. V. Salm. 2003. Resistance and Resilience to coral bleaching: Impications for Coarl Reef Conservation and Management. Conserv. Biol. 17 (4): 956-967. 